\title{
RESIDUE CLASSES OF LAGRANGIAN SUBBUNDLES AND MASLOV CLASSES
}

\author{
HARUO SUZUKI
}

\begin{abstract}
For Lagrangian subbundles with singularities in symplectic vector bundles, explicit formulas of relation between their residue classes and Maslov classes outside singularities are obtained. Then a Lagrangian subbundle with singularity is constructed where all possible Maslov classes are nonzero but residue classes vanish for dimension $>2$. Moreover, a Lagrangian immersion with singularity is constructed, where the similar property for the associated Maslov classes and residue classes is shown.
\end{abstract}

\section{INTRODUCTION}

Let $(E, \Omega)$ be a symplectic $2 n$-vector bundle over a differentiable manifold $M$ with a symplectic structure $\Omega$. $E$ has a complex operator $J$ compatible with $\Omega$. For two Lagrangian subbundles $L_{0}, L_{1}$ of $E$, Maslov classes $\mu_{h}\left(E, L_{0}, L_{1}\right) \in H^{4 h-3}(M ; \mathbf{R})$ are defined by I. Vaisman [V]. They are obstructions for $L_{0}, L_{1}$ to be transversal everywhere. Let $\Sigma$ be a closed set of $M$ and $L$ a Lagrangian subbundle of $\left.E\right|_{M \backslash \Sigma}$. We take $\Sigma$ as a set of singular points of the Lagrangian subbundle $L$. By making use of D. Lehmann's technique of [L1] and [L2], for odd Chern polynomials $c_{2 h-1}$, one can define $h$-residue classes $\operatorname{res}_{h}(L, \Sigma) \in H^{4 h-2}(M, M \backslash \Sigma ; \mathbf{R})$ on the singularity $\Sigma$ of the Lagrangian subbundle $L$, if $\Sigma$ has a neighborhood which is a submanifold with boundary and is contained in an open set retracting to $\Sigma$ by deformation. If $M$ is a differentiable oriented manifold of dimension $m$ and $\Sigma$ is compact, the Alexander-Lefschetz duality $\mathscr{D}$ yields homology classes $\operatorname{Res}_{h}(L, \Sigma)=\mathscr{D} \operatorname{res}_{h}(L, \Sigma) \in H_{m-4 h+2}(\Sigma ; \mathbf{R})$ on the singular set $\Sigma$.

Theorem 3.1. Let $E$ be a symplectic vector bundle over a differentiable manifold $M$ and $L$ a Lagrangian subbundle of $\left.E\right|_{M \backslash \Sigma}$ for a closed subset $\Sigma$ of $M$. Suppose that $U$ is an open set containing a submanifold with boundary which is a neighborhood of $\Sigma$, and retracts to $\Sigma$ by deformation. Let $L_{0}$ be a Lagrangian subbundle of $\left.E\right|_{U}, \mu_{h}\left(\left.E\right|_{U \backslash \Sigma},\left.L_{0}\right|_{U \backslash \Sigma},\left.L\right|_{U \backslash \Sigma}\right)$ Maslov classes on $\left.E\right|_{U \backslash \Sigma}$ and $\operatorname{res}_{h}(L, \Sigma)$ h-residue classes of $L$ on $\Sigma$. Then one obtains that

$$
\operatorname{res}_{h}(L, \Sigma)=e \circ \delta^{*} \mu_{h}\left(\left.E\right|_{U \backslash \Sigma},\left.L_{0}\right|_{U \backslash \Sigma},\left.L\right|_{U \backslash \Sigma}\right)
$$

where $\delta^{*}: H^{4 h-3}(U \backslash \Sigma ; \mathbf{R}) \rightarrow H^{4 h-2}(U, U \backslash \Sigma ; \mathbf{R})$ are connecting homomorphisms of cohomology exact sequence for the pair $(U, U \backslash \Sigma)$, and $e$ : $H^{4 h-2}(U, U \backslash \Sigma ; \mathbf{R}) \rightarrow H^{4 h-2}(M, M \backslash \Sigma ; \mathbf{R})$ are excision isomorphisms.

Received by the editors February 16, 1994.

1980 Mathematics Subject Classification (1985 Revision). Primary 57R20; Secondary 58F05. 
Let $\left(V, \Omega_{V}\right)$ be a symplectic $2 m$-manifold and $f: M \rightarrow V$ a map which is differentiable outside a closed set $\Sigma_{0} \subset M$. Moreover, we suppose that $\left.f\right|_{M \backslash \Sigma_{0}}$ is isotropic, that is $\left(\left.f\right|_{M \backslash \Sigma_{0}}\right)^{*} \Omega_{V}=0$. We set

$$
\Sigma=\left\{x \in M \backslash \Sigma_{0}\left|\operatorname{ker} f_{*}\right|_{x} \neq 0\right\} \cup \Sigma_{0} .
$$

The pullback $E_{f}=f^{*} T V$ of the tangent bundle $T V$ by $f$ has a natural symplectic structure $\Omega_{f}$ induced from $\Omega_{V}$ by $f$. The tangent bundle $L_{f}=T(M \backslash \Sigma)$ is a canonically embedded Lagrangian subbundle of the symplectic $2 m$-vector bundle $\left.E_{f}\right|_{M \backslash \Sigma}$. If $\Sigma$ has a neighborhood which is a submanifold with boundary and retracts to $\Sigma$ by deformation, then $h$-residue classes $\operatorname{res}_{h}(f, \Sigma)=\operatorname{res}_{h}\left(L_{f}, \Sigma\right) \in H^{4 h-2}(M, M \backslash \Sigma ; \mathbf{R})$ are defined (cf. Corollary 3.2).

We fix a complex operator $J$ of $E$ compatible to its symplectic structure. Let $U_{J}(E)$ be the $U(n)$-reduction of the principal $G(n, \mathbf{C})$ bundle associated with the complex $n$-vector bundle yielded from $E$ and $J$. Chern-Simons-Maslov classes $\mu^{h}(E, J, L) \in H^{4 h-3}\left(U_{J}(E) ; \mathbf{R}\right)$ are defined by [V].

Theorem 4.1. Let $E$ be a trivial symplectic $2 n$-vector bundle over a differentiable manifold $M$ with a trivializing section

$$
s: M \rightarrow U_{J}(E) \text {. }
$$

If $L$ is a Lagrangian subbundle of $\left.E\right|_{M \backslash \Sigma}$ for a closed set $\Sigma \subset M$ and if $\Sigma$ has a neighborhood which is a submanifold with boundary and retracts to $\Sigma$ by deformation, then we have

$$
\operatorname{res}_{h}(L, \Sigma)=s^{*} \mu^{h}\left(\left.E\right|_{M \backslash \Sigma}, J, L\right) .
$$

We construct a Lagrangian subbundle $L$ of a trivial symplectic vector bundle $D \times \mathbf{C}^{n}$ over a manifold $D$ with a nowhere dense singular set $\Sigma$. $D$ is the $\mathbf{R}^{2}$ vector bundle associated with the $S^{1}$-bundle $U(n) / O(n) \rightarrow U(n) / U(1) \cdot O(n)$ and $L$ is defined outside the zero section $\Sigma$ of $D$. Maslov classes of $L$ with respect to the "horizontal" Lagrangian subbundle $L_{0}$ of $D \times C^{n}$ are all nonzero. But as for residue classes of $L$ on $\Sigma$, one proves, by Theorem 3.1, the following,

Theorem 5.2. We have

$$
\operatorname{res}_{h}(L, \Sigma)= \begin{cases}\delta^{*} \Delta_{*} y_{1} \neq 0, & h=1 \\ 0, & h>1\end{cases}
$$

where $\delta^{*}: H^{1}(D \backslash \Sigma ; \mathbf{R}) \rightarrow H^{2}(D, D \backslash \Sigma ; \mathbf{R})$ is the connecting homomorphism of cohomology exact sequence for the pair $(D, D \backslash \Sigma)$ and $\Delta_{*} y_{1}$ (cf. [K-T, 6.28 Theorem]) is the canonical generator of the cohomology group $H^{1}(D \backslash \Sigma ; \mathbf{R}) \cong$ $H^{1}(U(n) / O(n) ; \mathbf{R})$.

Moreover one can construct a Lagrangian immersion $F: D \rightarrow \mathbf{C}^{\frac{n(n+1)}{2}+1}$ with a singular set $\Sigma_{\varepsilon}$ from the Lagrangian immersion $f: U(n) / O(n) \rightarrow \mathbf{C}^{\frac{n(n+1)}{2}}$ of J.-M. Morvan and L. Niglio [M-L]. Note that the singular set $\Sigma_{\varepsilon}$ contains interior point.

Theorem 7.2. Let $F: D \rightarrow \mathrm{C}^{\frac{n(n+1)}{2}+1}$ be the Lagrangian immersion with the singularity $\Sigma_{\varepsilon}$, stated in the above. Then we have h-residue classes

$$
\operatorname{res}_{h}\left(F, \Sigma_{\varepsilon}\right) \begin{cases}\neq 0, & h=1 \\ =0, & h>1\end{cases}
$$


and all Maslov classes for degree $<\frac{n(n+1)}{2}$ of $\left.F\right|_{D \backslash \Sigma_{\varepsilon}}$ with respect to the standard horizontal Lagrangian subbundle are nontrivial.

Contents of the present paper are as follows. In Section 1, we review Maslov classes and Chern-Simons-Maslov classes for Lagrangian subbundles. In Section 2 , we consider a connection with singularity on a principal bundle and review their $c_{2 h-1}$-residue classes through Čech-de Rham complex associated with the singularity. In Section 3, one defines $h$-residue classes $(h \geqq 1)$ of a Lagrangian subbundle on singularity and proves Theorem 3.1. The result is applied to singularities of Lagrangian immersions. In Section 4, we treat trivial symplectic $2 n$-vector bundle and Lagrangian subbundles with singularities. Then Theorem 4.1 is proved. In Section 5, we construct the example of Lagrangian subbundle with singularity and prove Theorem 5.2. In Section 6, for a Lagrangian immersion to a symplectic space from a manifold $N$ with $S^{1}$-bundle structure, a general construction of a Lagrangian immersion of the associated $\mathbf{R}^{2}$-bundle $D N$ to a symplectic space with singularity is made and then in the last section, Theorem 7.2 is proved.

\section{Maslov Classes}

Let $\pi_{E}: E \rightarrow M$ be a symplectic $2 n$-vector bundle over a differentiable manifold $M$ with a symplectic form $\Omega$. We choose a complex operator $J: E \rightarrow E$ compatible with $\Omega$ and regard $E$ as a complex $n$-vector bundle $(E, J)$. The principal $G L(n ; \mathbf{C})$-bundle associated with $E$ is reduced to a principal $U(n)$ bundle $\pi: U_{J}(E) \rightarrow M$. Let $L \subset E$ be a Lagrangian subbundle, i.e., a real $n$-dimensional subbundle with $\left.\Omega\right|_{L}=0$. Then the structural group of $E$ is reduced to $O(n)$ and one gets a principal $O(n)$-subbundle of $U_{J}(E)$, $\pi: U_{J}(E, L) \rightarrow M$. An $O(n)$-connection $\theta$ on $U_{J}(E, L)$ is extended on $U_{J}(E)$ naturally. The extended connection is called $L$-orthogonal unitary connection and is denoted by $\theta$ too. For the odd Chern polynomial $c_{2 h-1}$ and the curvature form $\Theta=d \theta+[\theta, \theta]$, we have

$$
c_{2 h-1}\left(\Theta^{(2 h-1)}\right)=0 \text {. }
$$

Let $\Delta(\theta)$ be the homomorphism of a $U(n)$-invariant polynomial algebra to the algebra of differentiable forms on $U_{J}(E)$ defined by substituting the curvature $\Theta$ of $\theta$. In particular, we have $\Delta(\theta) c_{k}=c_{k}\left(\Theta^{(k)}\right)$. Let $L_{0} \subset E$ be another Lagrangian subbundle. Then the principal $U(n)$-bundle $U_{J}(E)$ has another $O(n)$-reduction $U_{J}\left(E, L_{0}\right)$. Let $\theta_{0}$ be an $L_{0}$-orthogonal unitary connection and $\Delta^{1}$ a standard 1-simplex. For $t \in \Delta^{1}$,

$$
\hat{\theta}=(1-t) \theta_{0}+t \theta
$$

is a connection 1-form on the principal $U(n)$-bundle $U_{J}(E) \times \Delta^{1} \rightarrow M \times \Delta^{1}$. Let $\hat{\Theta}$ denote the curvature form of $\hat{\theta}$. (4h-3)-forms on $U_{J}(E)$ are defined by the formula

$$
\begin{aligned}
\Delta\left(\theta_{0}, \theta\right) c_{2 h-1} & =-\oint_{\Delta^{1}} c_{2 h-1}\left(\hat{\boldsymbol{\Theta}}^{(2 h-1)}\right) \\
& =(2 h-1) \int_{0}^{1} c_{2 h-1}\left(\theta-\theta_{0}, \hat{\Theta}_{t}^{(2 h-2)}\right) d t
\end{aligned}
$$


where $\oint_{\Delta^{1}}$ means an integration over the fiber of the $\Delta^{1}$-bundle $M \times \Delta^{1} \rightarrow M$, and

$$
\hat{\Theta}_{t}=t d\left(\theta-\theta_{0}\right)+\left[(1-t) \theta_{0}+t \theta,(1-t) \theta_{0}+t \theta\right] .
$$

Since $\theta$ and $\theta_{0}$ are $U(n)$-extensions of orthogonal connections, it follows that

$$
c_{2 h-1} \in \operatorname{Ker} \Delta\left(\theta_{0}\right) \cap \operatorname{Ker} \Delta(\theta),
$$

and hence we get

$$
d\left(\Delta\left(\theta_{0}, \theta\right) c_{2 h-1}\right)=\Delta(\theta) c_{2 h-1}-\Delta\left(\theta_{0}\right) c_{2 h-1}=0 .
$$

The $(4 h-3)$-forms $\Delta\left(\theta_{0}, \theta\right) c_{2 h-1}$ on $U_{J}(E)$ are adjoint invariant and so they are regarded as closed forms on the base space $M$. Their cohomology classes $\left[\Delta\left(\theta_{0}, \theta\right) c_{2 h-1}\right] \in H^{4 h-3}(M ; \mathbf{R})$ do not depend on the choices of the $L_{0^{-}}$ orthogonal unitary connection $\theta_{0}$ and the $L$-orthogonal unitary connection $\theta$. This fact is shown by making use of $\Delta^{1}$-bundle Stokes formula (cf. [V]). These cohomology classes are called Maslov classes of $\left(E, L_{0}, L\right)$ and are denoted by $\mu_{h}\left(E, L_{0}, L\right)$. Let $\theta$ be again an $L$-orthogonal unitary connection on $U_{J}(E)$. For $t \in \Delta^{1}, t \theta$ is a differential 1 -form on $U_{J}(E) \times \Delta^{1}$, which is not necessarily a connection on the principal $U(n)$-bundle $U_{J}(E) \times \Delta^{1} \rightarrow M \times \Delta^{1}$. We take a "formal curvature" of $t \theta$,

$$
\bar{\Theta}=d(t \theta)+[t \theta, t \theta]=d t \wedge \theta+\bar{\Theta}_{t}
$$

where $\bar{\Theta}_{t}$ is defined by

$$
\overline{\boldsymbol{\Theta}}_{t}=t d \theta+t^{2}[\theta, \theta]=t \Theta+t(t-1)[\theta, \theta] .
$$

A suspension form of the Chern form $\Delta(\theta) c_{2 h-1}$ is defined by

$$
\begin{aligned}
T(\theta) c_{2 h-1} & =-\oint_{\Delta_{1}} c_{2 h-1}\left(\bar{\Theta}^{(2 h-1)}\right) \\
& =(2 h-1) \int_{0}^{1} c_{2 h-1}\left(\theta, \bar{\Theta}_{t}^{(2 h-2)}\right) d t .
\end{aligned}
$$

By S.S. Chern and J. Simons [C-S], one obtains

$$
d\left(T(\theta) c_{2 h-1}\right)=\Delta(\theta) c_{2 h-1}=0
$$

The de Rham cohomology classes $\left[T(\theta) c_{2 h-1}\right] \in H^{4 h-3}\left(U_{J}(E) ; \mathbf{R}\right)$ do not depend on the choice of an $O(n)$-connection $\theta$, by the $\Delta^{1}$-bundle Stokes formula (cf. [V]). These cohomology classes are Chern-Simons-Maslov classes of $(E, J, L)$ and denoted by $\mu^{h}(E, J, L)$.

\section{RESIDUE CLASSES OF CONNECTION WITH SINGULARITY}

Let $M$ be a differentiable manifold of dimension $m, \Sigma$ a closed set of $M$ and $U$ an open neighborhood of $\Sigma$ admitting a deformation retraction $r: U \rightarrow \Sigma$. Let $W$ be a submanifold of dimension $m$ with boundary in $U$ such that $W \supset \Sigma, \partial W \cap \Sigma=\varnothing$ and, moreover, $\left.r\right|_{W}$ is a deformation retraction too for the sake of simplicity. Set $V=M \backslash \Sigma$. We review here D. Lehmann's definition of residue classes in [L2].

Remark. According to [L1], the condition of deformation retraction for $W$ is not necessary. 
A Mayer-Vietoris complex associated with $\mathscr{U}=\{V, U\}$ is the differential graded algebra

$$
\mathscr{R}^{*}(\mathscr{U})=\bigwedge^{*} V \oplus \bigwedge^{*} U \oplus \bigwedge^{*-1}(U \cap V)
$$

with the differential operator

$$
D(a, b, \xi)=\left(d a, d b,-d \xi+\left.b\right|_{U \cap V}-\left.a\right|_{U \cap V}\right)
$$

and with the product

$$
(a, b, \xi) \cup\left(a^{\prime}, b^{\prime}, \xi^{\prime}\right)=\left(a \wedge a^{\prime}, b \wedge b^{\prime},(-1)^{|a|} a \wedge \xi^{\prime}+\xi \wedge b^{\prime}\right)
$$

where $|a|=\operatorname{deg} a$. The natural inclusion map $\varepsilon: \Lambda^{*} M \rightarrow \mathscr{R}^{*}(\mathscr{U})$ defined by

$$
u \mapsto\left(\left.u\right|_{V},\left.u\right|_{U}, 0\right)
$$

induces an isomorphism of cohomology algebra by Weil Theorem [W], [B-T]. The projection $\mathscr{R}^{*}(\mathscr{U}) \rightarrow \Lambda^{*} V$ defined by

$$
(a, b, \xi) \mapsto a
$$

is a surjective homomorphism of differential graded algebras. Its kernel $\Lambda^{*} U \oplus$ $\bigwedge^{*-1}(U \cap V)$ has a cohomology algebra $H^{*}(M, M \backslash \Sigma ; \mathbf{R})$. By identifying $H_{*}(\Sigma ; \mathbf{R})$ with $\operatorname{Hom}\left(H_{D R}^{*}(U), \mathbf{R}\right)$, the map $\bigwedge^{m-k} U \oplus \bigwedge^{m-k-1}(U \cap V) \rightarrow$ $\operatorname{Hom}\left(\bigwedge^{k} U, \mathbf{R}\right)$ defined by

$$
(b, \xi) \mapsto\left(\gamma \mapsto \int_{W} b \wedge \gamma-\int_{\partial W} \xi \wedge \gamma\right)
$$

induces the Alexander-Lefschetz duality $\mathscr{D}: H^{m-k}(M, M \backslash \Sigma ; \mathbf{R}) \cong H_{k}(\Sigma ; \mathbf{R})$, if $M$ is oriented and $\Sigma$ is compact. Let $M, \Sigma$ and $U$ are as above. Usually we assume that Int $\Sigma=\varnothing$. Moreover, if $\Sigma$ is a differentiable submanifold, then one can take a tubular neighborhood of $\Sigma$ as $U$ with a deformation refraction $r: U \rightarrow \Sigma$. Let $(P, M, \pi, G)$ be a principal $G$-bundle where $G$ is a Lie group and $\theta$ a connection on $\left.P\right|_{M \backslash \Sigma}$ with $c_{k} \in \operatorname{Ker} \Delta(\theta)$. Let $\theta^{\prime}$ be an arbitrary connection on $\left.P\right|_{U}$. Then

$$
\left(\Delta\left(\theta^{\prime}\right) c_{k}, \Delta\left(\theta, \theta^{\prime}\right) c_{k}\right) \in \bigwedge^{2 k} U \oplus \bigwedge^{2 k-1}(U \cap V)
$$

are $2 k$-cocycles and their cohomology classes in $H^{2 k}(M, M \backslash \Sigma ; \mathbf{R})$ do not depend on the choice of $\theta^{\prime}$. It depends only on the $c_{k}$-homotopy class of $\theta$ (cf. [L2, pp. 94-95]), that is, if there is a connection $\varphi$ on the principal $G$ bundle $\left.P\right|_{M \backslash \Sigma} \times \Delta^{1} \rightarrow(M \backslash \Sigma) \times \Delta^{1}$ with $c_{k} \in \operatorname{Ker} \Delta(\varphi)$ and $\theta_{i}=\left.\varphi\right|_{\left.P\right|_{M \backslash \Sigma \times\{i\}}}$, $i=0,1$, then we have

$$
\left[\left(\Delta\left(\theta^{\prime}\right) c_{k}, \Delta\left(\theta_{0}, \theta^{\prime}\right) c_{k}\right)\right]=\left[\left(\Delta\left(\theta^{\prime}\right) c_{k}, \Delta\left(\theta_{1}, \theta^{\prime}\right) c_{k}\right)\right] .
$$

This is proved by making use of an affine combination of $\theta_{0}$ and $\theta_{1}$, and of the Stokes formula for trivial $\Delta^{1}$-bundles. The $c_{k}$-residue classes of $\theta$ on $\Sigma$ are the cohomology classes $\operatorname{res}\left(\theta, c_{k}\right)=\left[\Delta\left(\theta^{\prime}\right) c_{k}, \Delta\left(\theta, \theta^{\prime}\right) c_{k}\right] \in H^{2 k}(M, M \backslash \Sigma ; \mathbf{R})$ which are determined upto $c_{k}$-homotopy of the connection $\theta$. Note that we have excision isomorphisms

$$
H^{4 h-2}(U, U \backslash \Sigma ; \mathbf{R}) \cong H^{4 h-2}(M, M \backslash \Sigma ; \mathbf{R})
$$


and hence the $c_{k}$-residue classes are determined actually by a local data $\left.\theta\right|_{U \backslash \Sigma}$.

By the Alexander-Lefschetz duality, $\mathscr{D}: H^{2 k}(M, M \backslash \Sigma ; \mathbf{R}) \cong H_{m-2 k}(\Sigma ; \mathbf{R})$, one gets homology $c_{k}$-residue classes of $\theta$ on $\Sigma, \operatorname{Res}\left(\theta, c_{k}\right)=\mathscr{D} \operatorname{res}\left(\theta, c_{k}\right) \in$ $H_{m-2 k}(\Sigma ; \mathbf{R})$, if $M$ is oriented and $\Sigma$ is compact.

\section{LAGRANGIAN SUBBUNDLES WITH SINGULARITIES}

Let $\pi_{E}: E \rightarrow M$ be a symplectic $2 n$-vector bundle and $J: E \rightarrow E$ a compatible complex operator. Let $\Sigma$ be a closed set of $M$ and $L$ a Lagrangian subbundle of $\left.E\right|_{M \backslash \Sigma}$. $\Sigma$ is called a singular set of $L$. Let $U$ be an open set containing a submanifold with boundary which is a neighborhood of $\Sigma$ and retracts to $\Sigma$ by deformation. Let $\theta$ be an $L$-orthogonal unitary connection on $\left.U_{J}(E)\right|_{M \backslash \Sigma}$ and $\theta^{\prime}$ an arbitrary connection on $\left.U_{J}(E)\right|_{U}$. Since $c_{2 h-1} \in$ $\operatorname{Ker} \Delta(\theta)$, replacing $U$ by small one the $c_{2 h-1}$-residue classes of $\theta$ on $\Sigma$ are defined by

$$
\operatorname{res}\left(\theta, c_{2 h-1}\right)=\left[\left(\Delta\left(\theta^{\prime}\right) c_{2 h-1}, \Delta\left(\theta, \theta^{\prime}\right) c_{2 h-1}\right)\right] \in H^{4 h-2}(M, M \backslash \Sigma ; \mathbf{R}) .
$$

Any two $L$-orthogonal unitary connections on $\left.U_{J}(E)\right|_{M \backslash \Sigma}$ are $c_{2 h-1}$-homotopic by taking their affine combination, and therefore the $c_{2 h-1}$-residue classes $\operatorname{res}\left(\theta, c_{2 h-1}\right)$ of $\theta$ on $\Sigma$ do not depend on the choice of the connection $\theta$. They depend only on the Lagrangian subbundle $L$ on $M \backslash \Sigma$ and are denoted by $\operatorname{res}_{h}(L, \Sigma)$. We call them $h$-residue classes of $L$ on $\Sigma$. Suppose that $\left.E\right|_{U}$ has a Lagrangian subbundle $L_{0}$. Then one can take an $L_{0}$-orthogonal unitary connection on $\left.U_{J}(E)\right|_{U}$ as $\theta^{\prime}$ and obtain the following result.

Theorem 3.1. Let $\pi_{E}: E \rightarrow M$ be a symplectic vector bundle, and $L$ a Lagrangian subbundle of $\left.E\right|_{M \backslash \Sigma}$ for a closed set $\Sigma$ of $M$. Suppose that $U$ is an open set containing a submanifold with boundary which is a neighborhood of $\Sigma$, and retracts to $\Sigma$ by deformation. Let $L_{0}$ be a Lagrangian subbundle of $\left.E\right|_{U}, \mu_{h}\left(\left.E\right|_{U \backslash \Sigma},\left.L_{0}\right|_{U \backslash \Sigma},\left.L\right|_{U \backslash \Sigma}\right)$ Maslov classes on $\left.E\right|_{U \backslash \Sigma}$ and $\operatorname{res}_{h}(L, \Sigma)$ $c_{2 h-1}$-residue classes of $L$ on $\Sigma$. Then one obtains that

$$
\operatorname{res}_{h}(L, \Sigma)=e \circ \delta^{*} \mu_{h}\left(\left.E\right|_{U \backslash \Sigma},\left.L_{0}\right|_{U \backslash \Sigma},\left.L\right|_{U \backslash \Sigma}\right)
$$

where $\delta^{*}: H^{4 h-3}(U \backslash \Sigma ; \mathbf{R}) \rightarrow H^{4 h-2}(U, U \backslash \Sigma ; \mathbf{R})$ are connecting homomorphisms in the cohomology exact sequences of $(U, U \backslash \Sigma)$ and

$$
e: H^{4 h-2}(U, U \backslash \Sigma ; \mathbf{R}) \rightarrow H^{4 h-2}(M, M \backslash \Sigma ; \mathbf{R})
$$

are excision isomorphisms.

Proof. Since $\theta^{\prime}$ is a connection on $\left.U_{J}(E)\right|_{U}$, the $c_{2 h-1}$-residue classes $\operatorname{res}\left(\theta, c_{2 h-1}\right)$ have representative cocycles

$$
\left(\Delta\left(\theta^{\prime}\right) c_{2 h-1}, \Delta\left(\theta, \theta^{\prime}\right) c_{2 h-1}\right) \in \bigwedge^{4 h-2} U \oplus \bigwedge^{4 h-3}(U \backslash \Sigma),
$$

which represent $h$-residue classes $\operatorname{res}_{h}(L, \Sigma)$ of the Lagangian subbundle $L$ on its singular set $\Sigma$. On the other hand, since $\theta$ restricted to $U \backslash \Sigma$ is an $\left.L\right|_{U \backslash \Sigma^{-}}$ orthogonal connection on $\left.U_{J}(E)\right|_{U \backslash \Sigma}$ and $\theta^{\prime}$ is an $L_{0}$-orthogonal connection on $\left.U_{J}(E)\right|_{U}$, the Maslov classes $\mu_{h}\left(\left.E\right|_{U \backslash \Sigma},\left.L_{0}\right|_{U \backslash \Sigma},\left.L\right|_{U \backslash \Sigma}\right) \in H^{4 h-3}(U \backslash \Sigma ; \mathbf{R})$ are represented by

$$
\Delta\left(\theta, \theta^{\prime}\right) c_{2 h-1} \in \bigwedge^{4 h-3}(U \backslash \Sigma)
$$


which are identified with

$$
\left(\Delta\left(\theta, \theta^{\prime}\right) c_{2 h-1}, 0,0\right) \in \bigwedge^{4 h-3}(U \backslash \Sigma) \oplus \bigwedge^{4 h-3} U \oplus \bigwedge^{4 h-4}(U \backslash \Sigma) .
$$

We have

$$
\begin{aligned}
D\left(\Delta\left(\theta, \theta^{\prime}\right) c_{2 h-1}, 0,0\right) & =\left(d\left(\Delta\left(\theta, \theta^{\prime}\right) c_{2 h-1}\right), 0,-\Delta\left(\theta, \theta^{\prime}\right) c_{2 h-1}\right) \\
& =\left(0,0,-\Delta\left(\theta, \theta^{\prime}\right) c_{2 h-1}\right) \\
& =-\left(0, \Delta\left(\theta^{\prime}\right) c_{2 h-1}, \Delta\left(\theta, \theta^{\prime}\right) c_{2 h-1}\right)
\end{aligned}
$$

Therefore, it follows that

$$
\begin{aligned}
\delta^{*}\left[\Delta\left(\theta^{\prime}, \theta\right) c_{2 h-1}\right] & =-\delta^{*}\left[\Delta\left(\theta, \theta^{\prime}\right) c_{2 h-1}\right] \\
& =\left[\left(\Delta\left(\theta^{\prime}\right) c_{2 h-1}, \Delta\left(\theta, \theta^{\prime}\right) c_{2 h-1}\right)\right] .
\end{aligned}
$$

The excision map $e$ is the identity for the representative cocycles $\left(\Delta\left(\theta^{\prime}\right) c_{2 h-1}\right.$, $\left.\Delta\left(\theta, \theta^{\prime}\right) c_{2 h-1}\right)$ and so one gets the required formulas.

If $M$ is an oriented manifold of dimension $m$ and $\Sigma$ is compact, by the Alexander-Lefschetz duality $\mathscr{D}: H^{4 h-2}(M, M \backslash \Sigma ; \mathbf{R}) \stackrel{\cong}{\rightrightarrows} H_{m-4 h+2}(\Sigma ; \mathbf{R})$, one gets homology h-residue classes of $L$ on $\Sigma, \operatorname{Res}_{h}(L, \Sigma)=\mathscr{D} \operatorname{res}_{h}(L, \Sigma)$. Let $M$ be an $m$-dimensional manifold, $\left(V, \Omega_{V}\right)$ a symplectic $2 m$-manifold and $f: M \rightarrow V$ a map which is differentiable outside a closed set $\Sigma_{0} \subset M$. Moreover, we suppose that $\left.f\right|_{M \backslash \Sigma_{0}}$ is isotropic, that is $\left(\left.f\right|_{M \backslash \Sigma_{0}}\right)^{*} \Omega_{V}=0$. We set

$$
\Sigma=\left\{x \in M \backslash \Sigma_{0}\left|\operatorname{ker} f_{*}\right|_{x} \neq 0\right\} \cup \Sigma_{0} .
$$

The pullback $E_{f}=f^{*} T V$ of the tangent bundle $T V$ by $f$ has a natural symplectic structure $\Omega_{f}$ induced by $f$ from $\Omega_{V}$. The tangent bundle $L_{f}=$ $T(M \backslash \Sigma)$ is a canonically embedded Lagrangian subbundle of the symplectic $2 m$-vector bundle $\left.E_{f}\right|_{M \backslash \Sigma}$.

Corollary 3.2. Let $f: M \rightarrow\left(V, \Omega_{V}\right)$ be a map of an m-manifild to a symplectic $2 m$-manifold which is isotropic outside a closed set $\Sigma_{0}$. Suppose that the union $\Sigma$ of $\Sigma_{0}$ and the singular set of $f$ has a neighborhood which is a manifold with boundary and retracts to $\Sigma$ by deformation. Then h-residue classes $\operatorname{res}_{h}(f, \Sigma)=$ $\operatorname{res}_{h}\left(L_{f}, \Sigma\right) \in H^{4 h-2}(M, M \backslash \Sigma ; \mathbf{R})$ of $f$ are defined. Moreover if $E_{f}$ admits a Lagrangian subbundle $L_{0}$ then we have

$$
\operatorname{res}_{h}(f, \Sigma)=\delta^{*} \mu_{h}\left(\left.E_{f}\right|_{M \backslash \Sigma},\left.L_{0}\right|_{M \backslash \Sigma}, L_{f}\right) .
$$

Proof. We take the whole manifold $M$ as a neighborhood $U$ in Theorem 3.1, then the result follows immediately.

\section{Chern-Simons-Maslov CLASSES}

Suppose that the symplectic $2 n$-vector bundle $\pi_{E}: E \rightarrow M$ is trivial. Then the principal $U(n)$-bundle $U_{J}(E)$ associated with $E$ and a compatible complex operator $J$ has a section $s: M \rightarrow U_{J}(E)$ given by the trivialization. Let $\Sigma$ be a closed set of $M$ and $L$ a Lagrangian subbundle of $\left.E\right|_{M \backslash \Sigma}$. We are now interested in the cohomology classes $s^{*} \mu^{h}\left(\left.E\right|_{M \backslash \Sigma}, J, L\right) \in H^{4 h-3}(M \backslash \Sigma ; \mathbf{R})$. Let $L_{0}$ be a standard trivial Lagrangian subbundle of $E$ obtained by real part of the trivial complex $n$-vector bundle $(E, J)$. Now we get relations between $h$-residue class of $L$ on $\Sigma$ and Chern-Simons-Maslov classes of $L$ for $J$. 
Theorem 4.1. Let $E$ be a trivial symplectic $2 n$-vector bundle over $M$ with a trivializing section $s: M \rightarrow U_{J}(E)$ and $L_{0}$ the Lagrangian subbundle obtained from the trivialization. If $L$ is a Lagrangian subbundle of $\left.E\right|_{M \backslash \Sigma}$ for a closed set $\Sigma \subset M$ and if $\Sigma$ has a neighborhood which is a submanifold with boundary and retracts to $\Sigma$, then we have

$$
\begin{aligned}
\operatorname{res}_{h}(L, \Sigma) & =\delta^{*} \mu_{h}\left(\left.E\right|_{M \backslash \Sigma},\left.L_{0}\right|_{M \backslash \Sigma}, L\right) \\
& =\delta^{*} \circ s^{*} \mu^{h}\left(\left.E\right|_{M \backslash \Sigma}, J, L\right) .
\end{aligned}
$$

Proof. Let $\theta$ be an $L$-orthogonal unitary connection on $\left.U_{J}(E)\right|_{M \backslash \Sigma}$. Since $U_{J}(E)$ is a trivial $U(n)$-bundle, we have a projection to the fiber, $p_{f}: U_{J}(E) \rightarrow$ $U(n)$. Let $\omega$ denote the Maurer-Cartan form on $U(n)$ and set $\theta_{0}=p_{f}^{*} \omega$. Obviously $\theta_{0}$ is an $L_{0}$-orthogonal unitary connection. From the trivial bundle structure of $U_{J}(E)$, determined by the section $s$, it follows that

$$
\theta=\pi^{*} \circ s^{*} \theta+p_{f}^{*} \circ i^{*} \theta=\pi^{*} \circ s^{*} \theta+p_{f}^{*} \omega
$$

where $\pi^{*} s^{*} \theta$ is the $O(n)$-basic part of $\theta$ (cf. [Su, Lemma 3]) and $i$ is the inclusion map of $U(n)$ onto a fixed fiber. One obtains

$$
\begin{aligned}
\hat{\theta} & =(1-t) \theta_{0}+t \theta \\
& =(1-t) p_{f}^{*} \omega+t\left(\pi^{*} \circ s^{*} \theta+p_{f}^{*} \omega\right) \\
& =t \pi^{*} \circ s^{*} \theta+p_{f}^{*} \omega .
\end{aligned}
$$

Therefore we get

$$
s^{*} \hat{\theta}=s^{*}\left(t \pi^{*} \circ s^{*} \theta+p_{f}^{*} \omega\right)=s^{*} t \theta+s^{*} \circ p_{f}^{*} \omega=s^{*} t \theta,
$$

and hence

$$
s^{*} \hat{\Theta}=s^{*} \bar{\Theta}
$$

where $\hat{\Theta}$ is the curvature form of $\hat{\theta}$ and $\bar{\Theta}$ is the "curvature" form of $t \theta$.

It follows that

$$
\begin{aligned}
\mu_{h}\left(\left.E\right|_{M \backslash \Sigma},\left.L_{0}\right|_{M \backslash \Sigma}, L\right) & =\left[\Delta\left(\theta_{0}, \theta\right) c_{2 h-1}\right] \\
& =\left[-\oint_{\Delta^{1}} c_{2 h-1}\left(\hat{\boldsymbol{\Theta}}^{(2 h-1)}\right)\right] \\
& =\left[-\oint_{\Delta^{1}} c_{2 h-1}\left(s^{*} \hat{\Theta}^{(2 h-1)}\right)\right] \\
& =\left[-\oint_{\Delta^{1}} c_{2 h-1}\left(s^{*} \overline{\boldsymbol{\Theta}}^{(2 h-1)}\right)\right] \\
& =s^{*}\left[-\oint_{\Delta^{1}} c_{2 h-1}\left(\bar{\Theta}^{(2 h-1)}\right)\right] \\
& =s^{*}\left[T(\theta) c_{2 h-1}\right] \\
& =s^{*} \mu^{h}\left(\left.E\right|_{M \backslash \Sigma}, J, L\right) .
\end{aligned}
$$

On the other hand, by Theorem 3.1, we have

$$
\operatorname{res}_{h}(L, \Sigma)=\delta^{*} \mu_{h}\left(\left.E\right|_{M \backslash \Sigma},\left.L_{0}\right|_{M \backslash \Sigma}, L\right)
$$

and hence

$$
\operatorname{res}_{h}(L, \Sigma)=\delta^{*} \circ s^{*} \mu^{h}\left(\left.E\right|_{M \backslash \Sigma}, J, L\right) .
$$




\section{AN EXAMPLE OF LAGRANGIAN SUBBUNDLE}

We construct a Lagrangian subbundle with singularity in a symplectic vector bundle where only 1-residue class does not vanish but all Maslov classes outside the singularity are nontrivial. To do this, we use the Lagrangian Grassmannian manifold $U(n) / O(n)$.

Lemma 5.1. The manifold $U(n) / O(n)$ has an $S^{1}$-bundle structure with the structural group $S O(2)$.

Proof. Since we have a sequence of subgroups $O(n) \subset U(1) \cdot O(n) \subset U(n)$, one gets a fiber bundle

$$
\pi: U(n) / O(n) \rightarrow U(n) / U(1) \cdot O(n)
$$

whose fiber is $U(1) \cdot O(n) / O(n)$. But we have a natural diffeomorphism $U(1) / U(1) \cap O(n) \stackrel{\cong}{\rightarrow} U(1) \cdot O(n) / O(n)$ which maps $a \cdot(U(1) \cap O(n))$ to $a \cdot O(n)$ for each element $a \in U(1)$. Since we get

$$
U(1) / U(1) \cap O(n)=U(1) / O(1) \cong S^{1},
$$

it follows that $U(n) / O(n)$ is an $S^{1}$-bundle with the structural group $U(1) \equiv$ $S O(2)$.

Let $D$ be the $\mathbf{R}^{2}$-bundle associated with the $S^{1}$-bundle $U(n) / O(n) \rightarrow$ $U(n) / U(1) \cdot O(n)$ and consider the trivial $\mathrm{C}^{n}$-bundle

$$
E=D \times \mathbf{C}^{n} \rightarrow D \text {. }
$$

Let $L_{0}=D \times \mathbf{R}^{n}$ be the "horizontal" Lagrangian subbundle of $E$. For any nonzero vector $u$ of $D$, the normalized vector $\bar{u}=\frac{u}{\|u\|}$ is a point of $U(n) / O(n)$. Let $\Sigma$ be the zero section of $D$ and $L$ the Lagrangian subbundle of $\left.E\right|_{D \backslash \Sigma}$ defined by

$$
L_{u}=A_{\tilde{u}}\left(\mathbf{R}^{n}\right)
$$

where $A_{\bar{u}}$ is a unitary matrix representing the point $\bar{u} \in U(n) / O(n)$. Then Maslov classes for the Lagrangian subbundles $\left.L_{0}\right|_{D \backslash \Sigma}$ and $L$ in the symplectic vector bundle $\left.E\right|_{D \backslash \Sigma}$ is given by

$$
\mu_{h}\left(\left.E\right|_{D \backslash \Sigma},\left.L_{0}\right|_{D \backslash \Sigma}, L\right)=\Delta_{*} y_{2 h-1}
$$

where $\Delta_{*} y_{2 h-1}, h=1, \cdots, m, n=2 m$ or $2 m-1$ are canonical generators of the cohomology ring $H^{*}(U(n) / O(n) ; \mathbf{R})$ (cf. [K-T, 6.28 Theorem] and the proof of Theorem 4.1). Therefore all Maslov classes $\mu_{h}\left(\left.E\right|_{D \backslash \Sigma},\left.L_{0}\right|_{D \backslash \Sigma}, L\right)$ are nonzero. On the other hand, $h$-residue classes $\operatorname{res}_{h}(L, \Sigma)$ of $L$ on $\Sigma$ are computed as follows.

Theorem 5.2. Let $D$ be the $\mathbf{R}^{2}$-bundle associated with the $S O(2)$-bundle $U(n) / O(n) \rightarrow U(n) U(1) \cdot O(n)$, and $E=D \times \mathrm{C}^{n}$ the trivial symplectic vector bundle over $D$. Let $L$ be the canonical Lagrangian subbundle outside the zero section $\Sigma \cong U(n) / U(1) \cdot O(n)$. Then we have

$$
\operatorname{res}_{h}(L, \Sigma)= \begin{cases}\delta^{*} \Delta_{*} y_{1} \neq 0, & h=1, \\ 0, & h>1,\end{cases}
$$

where $\delta^{*}: H^{1}(D \backslash \Sigma ; \mathbf{R}) \rightarrow H^{2}(D, D \backslash \Sigma ; \mathbf{R})$ is the connecting homomorphism of the cohomology exact sequence for $(D, D \backslash \Sigma)$.

Proof. By Theorem 3.1 with $U=D$, we have

$$
\operatorname{res}_{1}(L, \Sigma)=\delta^{*} \mu_{1}\left(\left.E\right|_{D \backslash \Sigma},\left.L_{0}\right|_{D \backslash \Sigma}, L\right),
$$


since the excision isomorphism $H^{1}(D, D \backslash \Sigma ; \mathbf{R}) \cong H^{1}(U, U \backslash \Sigma ; \mathbf{R})$ is the identity map. The homomorphism $i^{*}: H^{1}(D ; \mathbf{R}) \rightarrow H^{1}(D \backslash \Sigma ; \mathbf{R})$ induced by the inclusion map $i: D \backslash \Sigma \rightarrow D$ is equivalent to the homomorphism $\pi^{*}$ : $H^{1}(U(n) / U(1) \cdot O(n) ; \mathbf{R}) \rightarrow H^{1}(U(n) / O(n) ; \mathbf{R})$ induced by the projection map $\pi: U(n) / O(n) \rightarrow U(n) / U(1) \cdot O(n)$. Since the space $U(n) / U(1) \cdot O(n)$ is simply connected, it follows that

$$
H^{1}(U(n) / U(1) \cdot O(n) ; \mathbf{R})=0 .
$$

Therefore $\delta^{*}$ is an injection and hence one gets

$$
\operatorname{res}_{1}(L, \Sigma)=\delta^{*} \Delta_{*} y_{1} \neq 0 .
$$

We set $N=U(n) / O(n)$ and $B=U(n) / U(1) \cdot O(n)$. The $E_{2}$-term of the cohomology spectral sequence (cf. [Se] and [M]) with real coefficients for the $S^{1}$-bundle $\pi: N \rightarrow B$ is

$$
\begin{aligned}
\sum E_{2}^{p, q} & =\sum H^{p}\left(B ; H^{q}\left(S^{1} ; \mathbf{R}\right)\right) \\
& =\sum H^{p}(B ; \mathbf{R}) \otimes H^{q}\left(S^{1} ; \mathbf{R}\right) .
\end{aligned}
$$

It is generated multiplicatively by the elements of $\sum E_{2}^{p, 0} \cong \sum H^{p}(B ; \mathbf{R})$ and the natural generator $u \in H^{1}\left(S^{1} ; \mathbf{R}\right) . E_{r}$ converges to $E_{\infty}=H^{*}(N ; \mathbf{R})$. Since we have $\Delta_{*} y_{1} \in H^{1}(N ; \mathbf{R})$ and $H^{1}(B ; \mathbf{R})=0, u$ have to stay alive to $E_{\infty}$. Therefore we get $d_{r} u=0$ for $r \geqq 2$. It is obvious that $d_{r}\left(E_{r}^{p, 0}\right)=0$ for $r \geqq 2$ and hence $E_{\infty}^{p, 0}=E_{2}^{p, q}$, that is,

$$
H^{*}(N ; \mathbf{R})=\sum H^{p}(B ; \mathbf{R}) \otimes H^{q}\left(S^{1} ; \mathbf{R}\right) .
$$

Now we obtain

$$
H^{k}(N ; \mathbf{R})=H^{k}(B ; \mathbf{R}) \oplus H^{k-1}(B ; \mathbf{R}) \otimes u .
$$

Therefore, primitive elements of $H^{k}(N ; \mathbf{R})$ for $k \geqq 2$ should belong to $H^{k}(B ; \mathbf{R})$ which is the subgroup of $\pi^{*}$-images. Since the homomorphisms $i^{*}$ : $H^{k}(D ; \mathbf{R}) \rightarrow H^{k}(D \backslash \Sigma ; \mathbf{R})$ induced by the inclusion map are equivalent to the homomorphisms $\pi^{*}$, primitve elements of $H^{k}(D \backslash \Sigma ; \mathbf{R}) \cong H^{k}(N ; \mathbf{R})$ for $k \geqq 2$ are $i^{*}$-images. Since $\Delta_{*} y_{2 h-1}$ are all primitive, they should be $i^{*}$-images for $h>1$ and hence one gets

$$
\operatorname{res}_{h}(L, \Sigma)=\delta^{*} \Delta_{*} y_{2 h-1}=0,
$$

for $h>1$.

\section{A LAGRANGIAN CONE OF LAGRANGIAN IMMERSION}

Let $N$ be an $S^{1}$-bundle over an $(n-1)$-dimensional manifold $B$ and $D N$ the $\mathbf{R}^{2}$-bundle associated with $N$. Obviously $N \subset D N$. Let $\left(\mathbf{R}^{2 n}, \Omega_{2 n}\right)$ be a canonical symplectic structure of $\mathbf{R}^{2 n}$ and $f: N \rightarrow \mathbf{R}^{2 n}$ a Lagrangian immersion. We construct a differentiable map $C f: D N \rightarrow \mathbf{R}^{2(n+1)}$ with singularity, which is a Lagrangian immersion outside a sufficiently small closed neighborhood $\Sigma_{\varepsilon}$ of the zero section of $D N$. Let $\varepsilon>0$ be a sufficiently small number and $\Sigma_{\varepsilon}$ the closed $\varepsilon$-disk bundle with the natural metric in the associated $\mathbf{R}^{2}$-bundle. The complement set $D N \backslash \Sigma_{\varepsilon}$ of $\Sigma_{\varepsilon}$ is obviously diffeomorphic to $N \times(\varepsilon, \infty)$. Any point $x \in D N$ is expressed as $x=t v$ with 
$v \in N, t \geqq 0$ where $t, v$ are unique outside the zero section. Define the map $D f: D N \rightarrow \mathbf{R}^{2 n} \times \mathbf{R}$ by

$$
D f(v, t)= \begin{cases}(f(v), t), & t \geqq \varepsilon \\ \left(\frac{t}{\varepsilon} f(v), t\right), & t<\varepsilon .\end{cases}
$$

$D f$ is an immersion outside $\Sigma_{\varepsilon}$. Let $i: \mathbf{R}^{2 n} \times \mathbf{R} \rightarrow \mathbf{R}^{2(n+1)}=\mathbf{R}^{2 n} \times \mathbf{R}^{2}$ be the canonical inclusion map defined by

$$
i(x, u)=(x, 0, u) .
$$

Note that the canonical symplectic structure $\Omega_{2(n+1)}$ is given by

$$
\Omega_{2(n+1)}=\pi_{\ell}^{*} \Omega_{2 n}+\pi_{r}^{*} \Omega_{2}
$$

where $\pi_{\ell}: \mathbf{R}^{2(n+1)} \rightarrow \mathbf{R}^{2 n}$ and $\pi_{r}: \mathbf{R}^{2(n+1)} \rightarrow \mathbf{R}^{2}$ are natural projections.

Lemma 6.1. Let $C f$ be the map $D f$ followed by i, i.e., $C f=i \circ D f: D N \rightarrow$ $\mathbf{R}^{2(n+1)}$. Then $\left.C f\right|_{D N \backslash \Sigma_{\varepsilon}}$ is a Lagrangian immersion and $\Sigma_{\varepsilon}$ is the singularity of the map $C f$ (in the sense that $C f$ is not an immersion or not isotropic there). Proof. For each point $x=(v, t) \in D N \backslash \Sigma_{\varepsilon}$, we have

$$
D f_{*}\left(T_{x} D N\right)=D f_{*}\left(T_{x}\left(D N \backslash \Sigma_{\varepsilon}\right)\right)=\left(f_{*} T_{v}(N), \mathbf{R}\right),
$$

and hence

$$
C f_{*}\left(T_{x}(D N)\right)=i_{*}\left(f_{*} T_{v}(N), \mathbf{R}\right)=\left(f_{*} T_{v}(N), 0, \mathbf{R}\right) .
$$

Since $f_{*} T_{v}(N)$ is a Lagrangian subspace of $\left(\mathbf{R}^{2 n}, \Omega_{2 n}\right), C f_{*}\left(T_{x}(D N)\right)$ is a Lagrangian subspace of $\left(\mathbf{R}^{2(n+1)}, \Omega_{2(n+1)}\right)$. Thus the map $C f: D N \rightarrow\left(\mathbf{R}^{2(n+1)}\right.$, $\left.\Omega_{2(n+1)}\right)$ is regarded as an isotropic map with the singularity $\Sigma_{\varepsilon}$.

The map $C f$ is called a Lagrangian cone of the Lagrangian immersion $f$. By Lemma 6.1, one can consider the Maslov classes associated with the Lagrangian immersion $\left.C f\right|_{D N \backslash \Sigma_{\varepsilon}}: D N \backslash \Sigma_{\varepsilon} \rightarrow\left(\mathbf{R}^{2(n+1)}, \Omega_{2(n+1)}\right)$ with respect to the trivial Lagrangian subbundle $L_{n+1,0}=\bigcup_{(x, y) \in \mathbf{R}^{2(n+1)}}\left(T_{x} \mathbf{R}^{n+1}, 0\right)$.

Lemma 6.2. Maslov classes of the Lagrangian immersion $\left.C f\right|_{D N \backslash \Sigma_{e}}$ with respect to the trivial Lagrangian subbundle $L_{n+1,0}$ coincide with those of the Lagrangian immersion $f: N \rightarrow\left(\mathbf{R}^{2 n}, \Omega_{2 n}\right)$ with respect to the trivial Lagrangian subbundle $L_{n, 0}$.

Proof. We take the trivial connection as an $L_{n, 0}$-orthogonal unitary connection $\theta_{n, 0}$ on $f^{*} T \mathbf{R}^{2 n}$. The tangent bundle $T N$ is a Lagrangian subbundle of $f^{*} T \mathbf{R}^{2 n}$, since $N$ is mapped by Lagrangian immersion $f$. Let $\theta_{n, 1}$ be a $T N$-orthogonal unitary connection on $f^{*} T \mathbf{R}^{2 n}$. The Maslov classes of $\left(f^{*} T \mathbf{R}^{2 n}, f^{*} L_{n, 0}, T N\right)$ are

$$
\mu_{h}\left(f^{*} T \mathbf{R}^{2 n}, f^{*} L_{n, 0}, T N\right)=\left[\Delta\left(\theta_{n, 0}, \theta_{n, 1}\right) c_{2 h-1}\right] \in H^{4 h-3}(N ; \mathbf{R})
$$

where $\Delta\left(\theta_{n, 0}, \theta_{n, 1}\right) c_{2 h-1}=f_{\Delta^{1}} c_{2 h-1}\left(\Theta^{(2 h-1)}\right)$ and $\Theta$ is the curvature form of the connection $\theta=(1-t) \theta_{n, 0}+t \theta_{n, 1}$ on $N \times \Delta^{1}$. We set $\alpha=\theta_{n, 1}-\theta_{n, 0}$ and denote the curvature of the connection $\left.\theta\right|_{t=\text { const }}$ by $\Theta_{t}$. Then it follows that

$$
\begin{aligned}
& \int_{\Delta^{1}} c_{2 h-1}\left(\Theta^{(2 h-1)}\right) \\
& \quad=(-1)^{h} \frac{\sqrt{-1}}{(2 \pi)^{2 h-1}(2 h-2) !} \int_{0}^{1}\left\{\Sigma \delta_{i_{1} \cdots i_{2 h-1}}^{j_{1} \cdots j_{2 h-1}} \alpha_{j_{1}}^{i_{1}} \wedge \Theta_{t, j_{2}}^{i_{2}} \wedge \cdots \wedge \Theta_{t, j_{2 h-1}}^{i_{2 h-1}}\right\} d t .
\end{aligned}
$$


Recall that $D N \backslash \Sigma_{\varepsilon}$ is diffeomorphic to $N \times(\varepsilon, \infty)$. Let $p_{\ell}: N \times(\varepsilon, \infty) \rightarrow N$ and $p_{r}: N \times(\varepsilon, \infty) \rightarrow(\varepsilon, \infty)$ be the left and right projection respectively. The Lagrangian subbundle $\left.T\left(D N \backslash \Sigma_{\varepsilon}\right) \subset C f^{*}\left(T \mathbf{R}^{2(n+1)}\right)\right|_{D N \backslash \Sigma_{\varepsilon}}$ is isomorphic to $p_{\ell}^{*} T N \oplus p_{r}^{*} T \mathbf{R}$. Since $p_{r}^{*} T \mathbf{R}$ is the trivial line bundle $\lambda$, the subbundle is denoted by $p_{\ell}^{*} T N \oplus \lambda$. Let $J_{n+1}$ and $g_{n+1}$ be the standard complex structure and the standard Hermitian metric on $\mathbf{R}^{2(n+1)}$. The pullback $C f^{*} J_{n+1}$ and $C f^{*} g_{n+1}$ is a compatible complex structure and a compatible Hermitian metric on $\left.C f^{*}\left(T \mathbf{R}^{2(n+1)}\right)\right|_{D N \backslash \Sigma_{e}}$. Let $\varphi$ be the trivial connection on $\lambda$. Then $\theta_{S, 1}=p_{\ell}^{*} \theta_{n, 1} \oplus \varphi$ is a $\left(p_{\ell}^{*} T N \oplus \lambda\right)$-orthogonal $U(n+1)$-connection on $\left.C f^{*}\left(T \mathbf{R}^{2(n+1)}\right)\right|_{D N \backslash \Sigma_{e}}$. The affine combination of $\theta_{n+1,0}$ and $\theta_{S, 1}$ is

$$
\begin{aligned}
\theta_{S} & =(1-t) \theta_{n+1,0}+t \theta_{S, 1} \\
& =(1-t)\left(p_{\ell}^{*} \theta_{n, 0} \oplus \varphi\right)+t\left(p_{\ell}^{*} \theta_{n, 1} \oplus \varphi\right) \\
& =p_{\ell}^{*}\left((1-t) \theta_{n, 0}+t \theta_{n, 1}\right) \oplus \varphi \\
& =p_{\ell}^{*} \theta \oplus \varphi,
\end{aligned}
$$

where the left projection $N \times \Delta^{1} \times(\varepsilon, \infty) \rightarrow N \times \Delta^{1}$ is denoted by $p_{\ell}$ too. Hence we have its curvature form

$$
\Theta_{S}=p_{\ell}^{*} \Theta \oplus 0=\left(p_{\ell}^{*} \alpha \wedge d t+p_{\ell}^{*} \Theta_{t}\right) \oplus 0
$$

since $\varphi$ is flat. Therefore one obtains

$$
\begin{aligned}
\Delta\left(\theta_{n+1,0}, \theta_{S, 1}\right) c_{2 h-1} & =-\oint_{\Delta^{1}} c_{2 h-1}\left(\Theta_{S}^{(2 h-1)}\right) \\
& =-\oint_{\Delta^{1}} c_{2 h-1}\left(\left(p_{\ell}^{*} \alpha \wedge d t+p_{\ell}^{*} \Theta_{t}\right)^{(2 h-1)}\right) \\
& =p_{\ell}^{*}\left(-\oint_{\Delta_{1}} c_{2 h-1}\left(\left(\alpha \wedge d t+\Theta_{t}\right)^{(2 h-1)}\right)\right) \\
& =p_{\ell}^{*}\left(-\Varangle_{\Delta^{1}} c_{2 h-1}\left(\Theta^{(2 h-1)}\right)\right) \\
& =p_{\ell}^{*}\left(\triangle\left(\theta_{n, 0}, \theta_{n, 1}\right) c_{2 h-1}\right) .
\end{aligned}
$$

Thus it follows that

$$
\begin{aligned}
& \mu_{h}\left(\left.C f^{*}\left(T \mathbf{R}^{2(n+1)}\right)\right|_{D \backslash \Sigma_{\varepsilon}},\left.C f^{*} L_{n+1,0}\right|_{D \backslash \Sigma}, T\left(D N \backslash \Sigma_{\varepsilon}\right)\right) \\
&=\left[\triangle\left(\theta_{n+1,0}, \theta_{S, 1}\right) c_{2 h-1}\right] \\
&=p_{\ell}^{*}\left[\triangle\left(\theta_{n, 0}, \theta_{n, 1}\right) c_{2 h-1}\right] \\
&=p_{\ell}^{*} \mu_{h}\left(f^{*} T \mathbf{R}^{2 n}, f^{*} L_{n, 0}, T N\right) .
\end{aligned}
$$

Note that $p_{\ell}^{*}: H^{4 h-3}(N, \mathbf{R}) \rightarrow H^{4 h-3}\left(D N \backslash \Sigma_{\varepsilon} ; \mathbf{R}\right)$ is an isomorphism since $p_{\ell}$ is the left projection $D N \backslash \Sigma_{\varepsilon}=N \times(\varepsilon, \infty) \rightarrow N$.

\section{AN EXAMPLE OF LAGRANGIAN IMMERSION WITH SINGULARITY}

We apply the construction of Lagrangian cone of a Lagrangian immersion to that of the Lagrangian Grassmannian manifold $U(n) / O(n)$ to the complex space $\mathbf{C}^{\frac{n(n+1)}{2}}$ given by J.-M. Morvan and L. Niglio [M-N]. Let $\mathbf{V}$ be the vector space of $(n \times n)$-complex symmetric matrices, which is identified with $\mathbf{C}^{\frac{n(n+1)}{2}} . \mathbf{V}$ has a natural Hermitian structure defined by $\langle X, Y\rangle=\operatorname{tr}(X \bar{Y})$ for $X, Y \in \mathbf{V}$. 
The map of $U(n)$ to $\mathbf{V}$ defined by $A \mapsto A^{t} A$ induces a map $f: U(n) / O(n) \rightarrow$ $\mathbf{V}$ from the quotient space by $O(n) . f$ is a Lagrangian immersion and its Maslov classes for degree $<\frac{n(n+1)}{2}$ with respect to the standard horizontal Lagrangian subbundle are all nonzero. Note that the complex space $C^{\frac{n(n+1)}{2}}$ is identified with the standard symplectic space $\left(\mathbf{R}^{n(n+1)}, \Omega_{n(n+1)}\right)$. Let $D$ denote the $\mathbf{R}^{2}$-bundle associated with the $S^{1}$-bundle $U(n) / O(n) \rightarrow U(n) / U(1)$. $O(n)$ as in Section 5 , and $\Sigma_{\varepsilon}$ the closed $\varepsilon$-disk bundle in $D$, which is a closed neighborhood of the zero section of $D$. By Lemma 6.1, one gets a map

$$
F=C f: D \rightarrow \mathbf{R}^{n(n+1)+2}
$$

such that $\left.F\right|_{D \backslash \Sigma_{\varepsilon}}$ is a Lagrangian immersion and $\Sigma_{\varepsilon}$ is the singularity of $F$. By Lemma 6.2, Maslov classes of the Lagrangian immersion $\left.F\right|_{D \backslash \Sigma_{e}}$ : $D \backslash \Sigma_{\varepsilon} \rightarrow\left(\mathbf{R}^{n(n+1)+2}, \Omega_{n(n+1)+2}\right)$ with respect to the standard horizontal Lagrangian subbundle $L_{\frac{n(n+1)}{2}+1,0}$ coincide with those of the Lagrangian immersion $f: U(n) / O(n) \rightarrow \mathbf{C}^{\frac{n(n+1)}{2}}=\left(\mathbf{R}^{n(n+1)}, \Omega_{n(n+1)}\right)$ with respect to the standard horizontal Lagrangian subbundle $L_{\frac{n(n+1)}{2}}, 0$.

Lemma 7.1. The Maslov classes for degree $<\frac{n(n+1)}{2}$ of the Lagrangian immersion $\left.F\right|_{D \backslash \Sigma_{e}}$ with respect to the standard horizontal Lagrangian subbundle $L_{\frac{n(n+1)}{2}+1,0}$ are all nonzero.

Proof. Since the Maslov classes for degree $<\frac{n(n+1)}{2}$ of the Lagrangian immersion $f$ with respect to the subbundle $L_{\frac{n(n+1)}{2}}, 0$ are all nonzero by [M-N], and since the Maslov classes of the Lagrangian immersion $\left.F\right|_{D \backslash \Sigma_{\varepsilon}}: D \backslash \Sigma_{\varepsilon} \rightarrow$ $\left(\mathbf{R}^{n(n+1)+2}, \Omega_{n(n+1)+2}\right)$ with respect to the subbundle $L_{\frac{n(n+1)}{2}, 0}$ coincide with those of $f$, the result follows.

$h$-residue classes of a Lagrangian immersion $f: M \rightarrow V$ with singularity $\Sigma$ are defined by $\operatorname{res}_{h}(f, \Sigma)=\operatorname{res}_{h}(T(M \backslash \Sigma), \Sigma)$ from Corollary 3.2. Now we look at the $h$-residue classes of the Lagrangian immersion $F: D \rightarrow C^{\frac{n(n+1)}{2}+1}$ with the singularity $\Sigma_{\varepsilon}$. Note that $\Sigma_{\varepsilon}$ is a small closed tubular neighborhood of the zero section of $D$ and hence contains interior points.

Theorem 7.2. Let $F: D \rightarrow C^{\frac{n(n+1)}{2}+1}$ be the Lagrangian immersion with the singularity $\Sigma_{\varepsilon}$, stated in the above. Then we have

$$
\operatorname{res}_{h}\left(F, \Sigma_{\varepsilon}\right) \begin{cases}\neq 0, & h=1, \\ =0, & h>1,\end{cases}
$$

and all Maslov classes for degree $<\frac{n(n+1)}{2}$ of $\left.F\right|_{D \backslash \Sigma_{e}}$ with respect to the standard horizontal Lagrangian subbundle are nontrivial.

Proof. By Corollary 3.2, we have

$$
\operatorname{res}_{h}\left(F, \Sigma_{\varepsilon}\right)=\delta^{*} \mu_{h}\left(\left(\left.F\right|_{D \backslash \Sigma_{\varepsilon}}\right)^{*} T \mathbf{R}^{n(n+1)+2}, T\left(D \backslash \Sigma_{\varepsilon}\right),\left(\left.F\right|_{D \backslash \Sigma_{\varepsilon}}\right)^{*} L_{\frac{n(n+1)}{2}+1,0}\right) .
$$

By Lemma 7.1, the Maslov classes in the right-hand side are all nonzero. By the same argument on the homomorphism

$$
\delta^{*}: H^{4 h-3}\left(D \backslash \Sigma_{\varepsilon} ; \mathbf{R}\right) \rightarrow H^{4 h-2}\left(D, D \backslash \Sigma_{\varepsilon} ; \mathbf{R}\right)
$$

as in the proof of Theorem 5.2, the result follows. 


\section{REFERENCES}

[B-T] R. Bott and L. W. Tu, Differential forms in algebraic topology, Springer, Berlin and New York, 1982.

[C-S] S. S. Chern and J. Simons, Characteristic forms and geometric invariants, Ann. of Math. (2) 99 (1974), 48-69.

[K-T] F. Kamber and $\mathrm{Ph}$. Tondeur, Foliated bundles and characteristic classes, vol. 493, Lecture Notes in Math., Springer, Berlin and New York, 1975.

[L1] D. Lehmann, Variété stratifiées $C^{\infty}$ : Intégration de Čech-de Rham, et théorie de Chern-Weil, Geometry and Topology of Submanifolds, II (Avignon, 1988), World Scientific, Teaneck, NJ, 1990, pp. 205-248.

[L2] D. Lehmann, Classes caractéristiques residuelles, Differential Geometry and Its Applications, World Scientific, Singapore, 1990, pp. 85-108.

[M] J. McCleary, User's guide to spectral sequences, Publish or Perlish, Wilmington, DE, 1985.

[M-N] J.-M. Morvan and L. Niglio, Classes caracteristiques des couples de sous-fibrés Lagrangiens, Ann. Inst. Fourier Grenoble 36 (1986), 193-209.

[Se] J.-P. Serre, Homologie singulière des espaces fibrés, Ann. of Math. 54 (1951), 425-505.

[Su] H. Suzuki, Chern-Simons-Malsov classes of some symplectic vector bundles, Proc. Amer. Math. Soc. 117 (1993), 541-546.

[V] I. Vaisman, Symplectic geometry and secondary characteristic classes, Birkhäuser, Boston and Basel, 1987.

[W] A. Weil, Sur les théorèmes de de Rham, Comment. Math. Helv. 26 (1952), 119-145.

Department of Mathematics, Hokkaido University, SAPPoro 060, JAPAN Current address: 7-3-5 Minamimachi, Makomanai, Minami-Ku, Sapporo 005, Japan

E-mail address: suzuki@math.hokudai.ac.jp 